\title{
Acute Headache Due to Intracerebral Hemorrhage Secondary to Brain Metastases
}

\author{
Zachary J. Cohen-Neamie ${ }^{1}$, Latha Ganti ${ }^{2,3,4,5}$, Thor S. Stead ${ }^{6}$, Joshua Walker ${ }^{4,2,} 7$, Frank Fraunfelter ${ }^{4}$,
} 2,8

1. Emergency Medicine, Trinity Preparatory School, Maitland, USA 2. Emergency Medicine, Envision Physician Services, Plantation, USA 3. Emergency Medicine, University of Central Florida College of Medicine, Orlando, USA 4. Emergency Medicine, Ocala Regional Medical Center, Ocala, USA 5. Emergency Medicine, HCA Healthcare Graduate Medical Education Consortium Emergency Medicine Residency Program of Greater Orlando, Olrando, USA 6. Medicine, Warren Alpert Medical School, Providence, USA 7. Emergency Medicine, University of Central Florida, Orlando, USA 8. Emergency Medicine, University of Central Florida, Providence, USA

Corresponding author: Latha Ganti, latha.ganti@ucf.edu

\begin{abstract}
Intracerebral hemorrhage (ICH) is a relatively common condition seen throughout the world, with the vast majority of cases referring to primary ICH. However, secondary ICH from other underlying conditions is also possible. In the present case, the patient presented with severe headaches. An initial computed tomography (CT) was taken which showed hyperdense regions in both the occipital lobe and right lateral ventricle. The patient was hypertensive upon arrival, so medication was given to lower his blood pressure. Due to the patient's history of hypertension, it was believed to be a case of primary ICH caused by high blood pressure, but because of the odd positioning of the hemorrhaging, it was recommended for magnetic resonance imaging (MRI) and angiography (MRA) to be taken. Using the MRI and MRA, it was found out that growing nodes were responsible for the hypodense regions on the CT. Considering the patient's history of renal cell carcinoma metastasizing to the abdomen and lungs, the nodes were diagnosed as brain metastasis (BM) developed from the patient's past kidney cancer. Considering the hemorrhaging locations in the brain, it was concluded that the ICH was secondary to BM. After consulting neurosurgery and hematology, the patient was discharged to his family. Although not very prevalent in cases of ICH, BM is a cause that can not be overlooked. Sometimes initial imaging does not reveal such an underlying source. It is always important to pay close attention to the characteristics of the ICH so that it is possible to determine the true reason for the hemorrhage.
\end{abstract}

Review began 07/26/2021 Review ended 07/26/2021 Published 08/04/2021

\section{(๑) Copyright 2021}

Cohen-Neamie et al. This is an open access article distributed under the terms of the Creative Commons Attribution License CC-BY 4.0., which permits unrestricted use, distribution, and reproduction in any medium, provided the original author and source are credited.
Categories: Neurology, Neurosurgery, Hematology

Keywords: renal cell carcinoma, brain metastases, intracerebral hemorrhage, headache, abnormal computed tomography

\section{Introduction}

Intracerebral hemorrhage (ICH) is any type of bleeding that occurs in the brain tissue. More than 1 million people around the world are diagnosed with ICH annually [1]. The most common cause of ICH is hypertension, due to cerebrovascular damage to small arteries and arterioles [2]. Other causes include arteriovenous malformations, amyloid angiopathy, and trauma [3]. Hemorrhage associated with brain metastases (BM) is an uncommon cause of ICH. They can either appear as the presenting symptom of BM, or can be found after the BM was already discovered [4]. Hemorrhage resulting from brain tumors can occur in up to $10 \%$ of all primary metastatic tumors [2]. ICH is also associated with secondary metastatic brain tumors, and accounts for $2 \%-7 \%$ of all spontaneous development of $\mathrm{ICH}[5,6]$. The authors present the case of a man with a history of clear cell renal carcinoma who complained of a severe temporal headache and was found to have ICH secondary to an occipital lobe mass.

\section{Case Presentation}

A 57-year-old right-handed male presented to the emergency department (ED) with acute headache. He began having severe headaches, dizziness, nausea, neck pain, and binocular blurry vision, and photophobia a few days prior. The headache was located in the right temporal region, and upon standing or moving his head to the right, the patient experienced dizziness. His past medical history included hypertension, hyperlipidemia, and clear cell renal carcinoma with metastasis to the lungs and abdomen. His past surgical history included a nephrectomy for his stage 2 renal cell carcinoma. The patient reported he drank six beers daily and was a former smoker. The last time the patient drank was five days prior. The patient denied ever taking any illicit drugs.

His initial vital signs included a blood pressure of $172 / 99 \mathrm{mmHg}$, a temperature of $97.2^{\circ} \mathrm{F}$, a pulse of 86 beats per minute, an oxygen saturation of $99 \%$ on room air, and a respiratory rate of 18 breaths per minute.

Physical examination revealed a well-nourished and well-developed male, who appeared his stated age. His 


\section{Cureus}

body-mass-index was $32.4 \mathrm{~kg} / \mathrm{m} 2$. The patient did not have any acute respiratory distress. The patient was awake, alert, and oriented to person, place, time, and situation. His speech was clear. His pupils were equal, round, and reactive to light. Extraocular eye movements were intact, although the patient did have blurry vision in the right lower quadrant of both eyes. His face was symmetrical, and the tongue was midline with no fasciculations or trauma. Sensation was intact to light touch and pinprick. His coordination exam revealed normal finger-nose-finger testing and normal heel-to-shin testing. Patient had 5/5 motor strength of his bilateral upper and lower extremities. His National Institutes if Health Stroke Scale (NIHSS) score was 0 .

His laboratory evaluation showed slight anemia with a hemoglobin of $12.5 \mathrm{~g} / \mathrm{dL}$, an elevated sedimentation rate of $100 \mathrm{~mm} / \mathrm{hr}$. All other laboratory evaluation was within normal limits.

Non-contrast computed tomography (CT) scan of the brain demonstrated moderate hemorrhage within the right ventricle. A $2.1 \mathrm{~cm}$ focal hypodensity in the left medial occipital knob was noted as was a mass within the posterior right ventricle at the area of the hemorrhage (Figure 1).

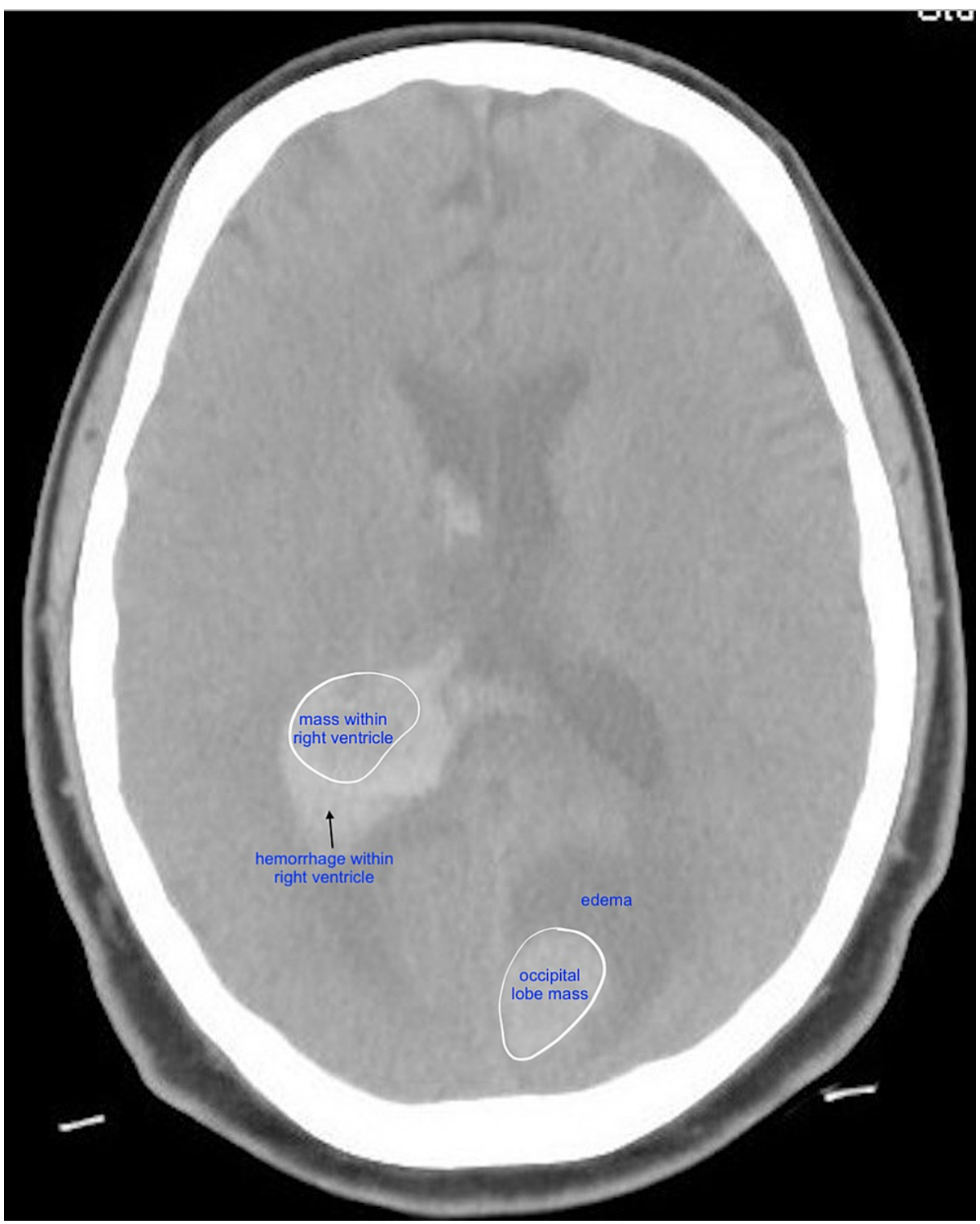

FIGURE 1: Non-contrast computed tomography (CT) scan demonstrating a $2 \mathrm{~cm}$ focal hyperdensity in the medial left occipital lobe, with appearance of intraparenchymal hemorrhage but suspicious for an underlying mass given the surrounding edema.

Considering the presence of edema surrounding the hyperdense regions, magnetic resonance imaging (MRI) and angiography (MRA) were ordered. MRA of the head was unremarkable. The MRI demonstrated an 


\section{Cureus}

enhancing nodule in the left occipital lobe with associated parenchymal hemorrhage and surrounding edema and an enhancing nodule in the right cord plexus also with associated right intraventricular hemorrhage and surrounding parenchymal edema, likely due to metastatic disease (Figure 2).

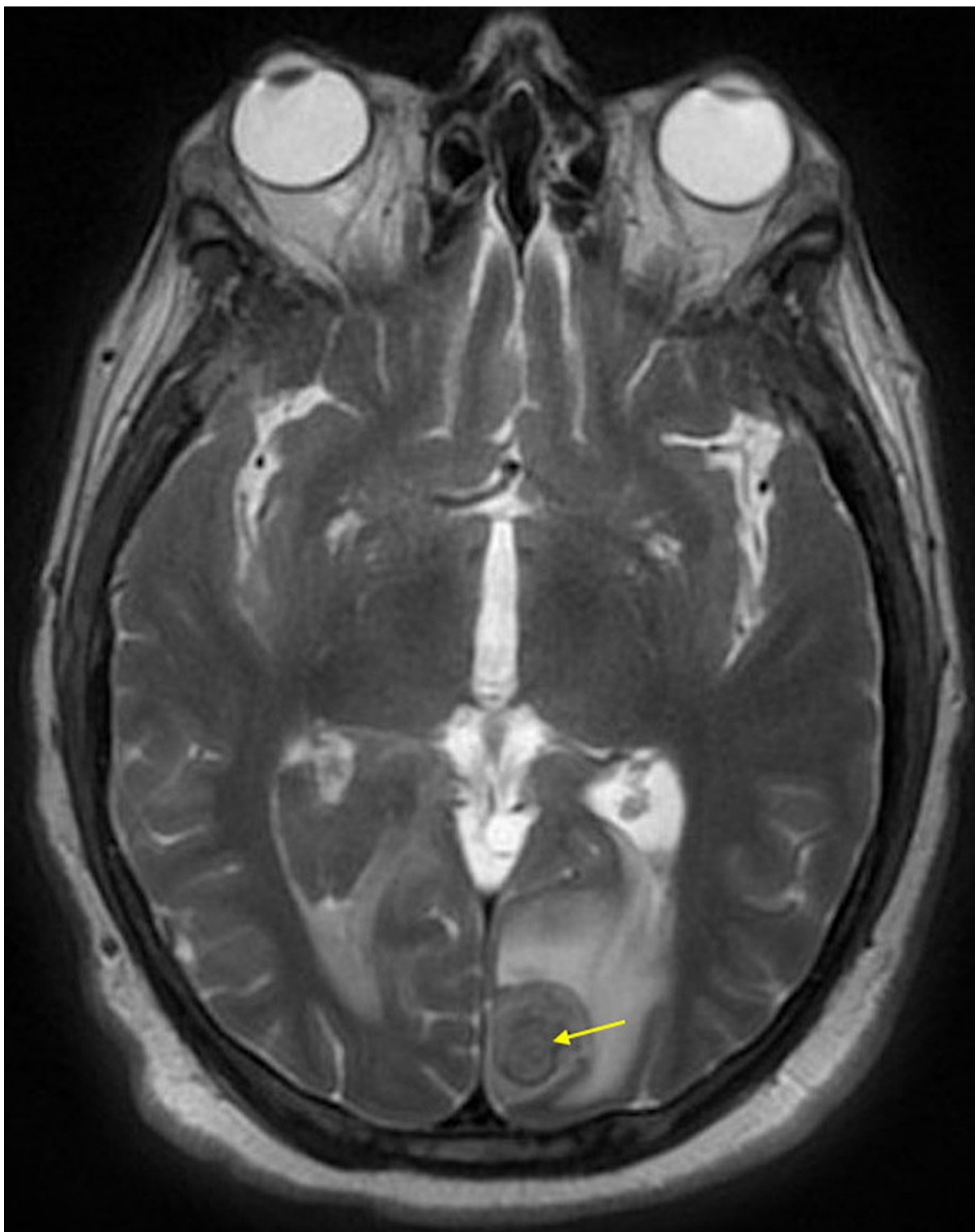

FIGURE 2: Brain magnetic resonance imaging (MRI) demonstrating focus of acute parenchymal hemorrhage in the left occipital lobe measuring $2.4 \times 1.8 \times 1.8 \mathrm{~cm}$ and an enhancing nodule in that region measuring $1.1 \times 1.1 \mathrm{~cm}$ (arrow).

The patient was given intravenous dexamethasone for the edema associated with the occipital lobe tumor, and nicardipine infusion for blood pressure control. Repeat CT scan the following day revealed stable appearance of the right lateral ventricular hemorrhage extending into the occipital and temporal horns; and stable appearance of the $2 \mathrm{~cm}$ focal hyperdensity in the medial aspect of the left occipital lobe with moderate vasogenic edema (Figure 3). 


\section{Cureus}
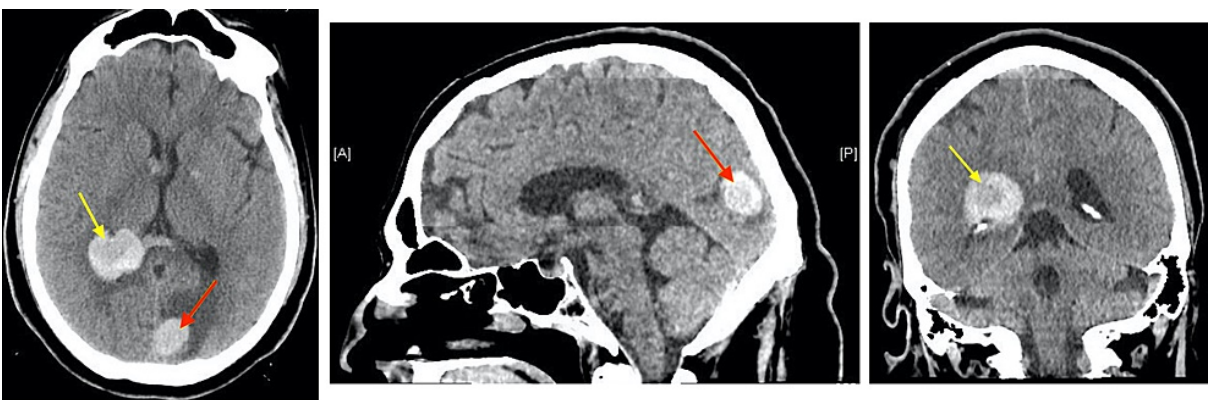

FIGURE 3: Axial, sagittal, and coronal computed tomography (CT) views demonstrating a $2 \mathrm{~cm}$ hyperdense lesion in the medial aspect of the left occipital lobe with moderate surrounding vasogenic edema (red arrow) and right lateral ventricular hemorrhage extending into the occipital and temporal horns (yellow arrow).

After being evaluated by neurology, neurosurgery, and hematology, his headache improved and given the paucity of his neurologic impairment, the patient was discharged home to the care of his family.

\section{Discussion}

The differential diagnosis of ICH from metastasis versus primary ICH can be challenging [7-9]. Clues to the former include atypical location of the ICH, non-hemorrhagic tissue within the ICH, multiple sites of ICH, and an uneven distribution of density within the ICH [10]. Edema surrounding the ICH is suspicious for an underlying cerebral metastasis, as is an indentation of the hematoma's surface [11].

In our patient, initially, the ICH was thought to be due to hypertension, as the patient had a history of uncontrolled blood pressure. However, the ICH was in two locations, which is unusual [12]. It was seen in the lateral ventricle as well as in the occipital region and was a sizable volume. If this ICH were due to a vascular cause, one would expect the neurologic findings to be quite dramatic; however, the patient's neurological exam was remarkably benign with an NIHSS score of 0 . Furthermore, the edema surrounding the area of hemorrhage was also suspicious for ICH of a non-vascular origin. The MRI elucidated the culprit to be a brain mass. BM are the most frequent cerebral tumors in adults with approximately $80 \%$ of the metastases located in the cerebral hemispheres and 20\% in the posterior fossa structures [13] (Figure 4). 


\section{Cureus}

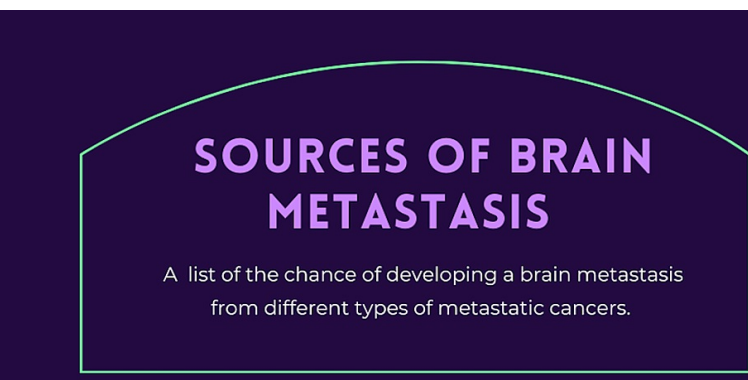

\section{LUNG CANCER \\ $50-60 \%$}
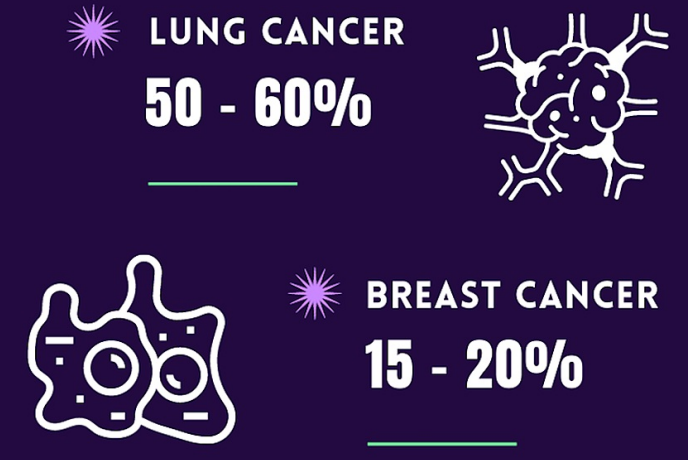

\section{BREAST CANCER}

$15-20 \%$

\section{缣 MELANOMA \\ $5-10 \%$}
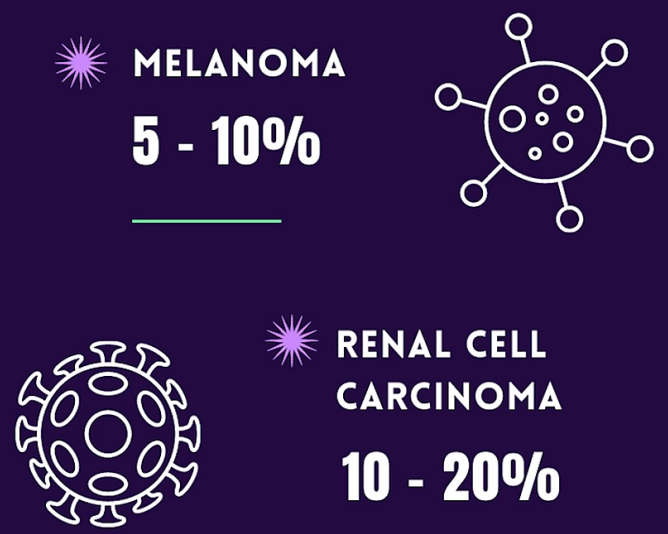

RENAL CELL

CARCINOMA

$10-20 \%$

\section{榙 GASTROINTESTINAL CANCER \\ $4-6 \%$}

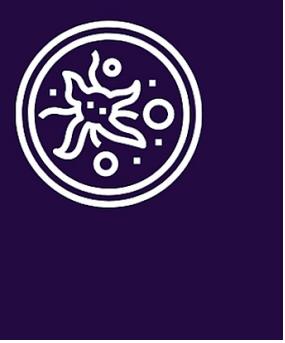

FIGURE 4: Infographic summarizing sources of brain metastases.

In our patient, the primary cancer was renal cell carcinoma, and his headache presentation revealed the metastases.

ICH is a feared complication of BM. In a cohort of 145,225 hospitalized patients with BM, ICH was present in $2.85 \%$, and the two most common underlying primary tumors were melanoma, and kidney cancer [5]. In a prospective series of 2041 patients with intracranial neoplasms and 692 patients with spontaneous ICH, the frequency of ICH in patients with intracranial neoplasms was $2.4 \%$. while $7.2 \%$ in the ICH group had tumorrelated ICH [6]. In an institutional cohort of 158 patients undergoing evaluation for BM due to renal cell carcinoma (RCC), 94.4\% had clear-cell RCC, and 90.6\% had extracranial metastases at diagnosis [14]. Our patient also had clear cell RCC and presented with known lung metastases. Patients with a solitary brain lesion (versus multiple) were significantly less likely to develop recurrence, and had a median recurrencefree time of 12.4 months. Many patients with cancer are on anticoagulants due to the increased prevalence 
of deep venous thrombosis in the population. This poses an additional risk for ICH secondary to metastases [15]. Fortunately, this was not the case with our patient.

\section{Conclusions}

Hemorrhagic BM is not a common etiology for ICH, and initial imaging may not reveal the etiology clearly. It is important to carefully consider the features of the ICH on imaging and obtain further studies if an underlying malignancy is suspected. Even considering the rarity of ICH secondary to BM, it should not be immediately disaffirmed. Improper diagnosis of hemorrhaging relating to metastatic cancer may lead to unnecessary interventions which could further worsen the situation.

\section{Additional Information \\ Disclosures}

Human subjects: Consent was obtained or waived by all participants in this study. NA issued approval NA. This research was supported (in whole or in part) by HCA Healthcare and/or an HCA Healthcare affiliated entity. The views expressed in this publication represent those of the author(s) and do not necessarily represent the official views of HCA Healthcare or any of its affiliated entities. . Conflicts of interest: In compliance with the ICMJE uniform disclosure form, all authors declare the following: Payment/services info: All authors have declared that no financial support was received from any organization for the submitted work. Financial relationships: All authors have declared that they have no financial relationships at present or within the previous three years with any organizations that might have an interest in the submitted work. Other relationships: All authors have declared that there are no other relationships or activities that could appear to have influenced the submitted work.

\section{Acknowledgements}

This research was supported (in whole or in part) by HCA Healthcare and/or an HCA Healthcare affiliated entity. The views expressed in this publication represent those of the author(s) and do not necessarily represent the official views of HCA Healthcare or any of its affiliated entities.

\section{References}

1. Shaheed TA, Glover N, Alboiny S: Nontraumatic spontaneous bilateral basal ganglia hemorrhage: a rare case report. Cureus. 2020, 12:e11299. 10.7759/cureus.11299

2. Fogelholm R, Eskola K, Kiminkinen T, Kunnamo I: Anticoagulant treatment as a risk factor for primary intracerebral haemorrhage. J Neurol Neurosurg Psychiatry. 1992, 55:1121-4. 10.1136/jnnp.55.12.1121

3. Fewel ME, Thompson BG Jr, Hoff JT: Spontaneous intracerebral hemorrhage: a review . Neurosurg Focus. 2003, 15:E1.

4. Lieu AS, Hwang SL, Howng SL, Chai CY: Brain tumors with hemorrhage. J Formos Med Assoc. 1999, 98:3657.

5. Lee V, Jairam V, Yu JB, Park HS: Nationwide patterns of hemorrhagic stroke among patients hospitalized with brain metastases: influence of primary cancer diagnosis and anticoagulation. Sci Rep. 2020, 10:10084. 10.1038/s41598-020-67316-8

6. Schrader B, Barth H, Lang EW, Buhl R, Hugo HH, Biederer J, Mehdorn HM: Spontaneous intracranial haematomas caused by neoplasms. Acta Neurochir (Wien). 2000, 142:979-85. 10.1007/s007010070052

7. Jouary T, Delaunay M, Taieb A: Hematoma-like metastases. J Am Acad Dermatol. 2006, 55:1106-7. 10.1016/j.jaad.2005.10.023

8. Mandybur TI: Intracranial hemorrhage caused by metastatic tumors . Neurology. 1977, 27:650-5. 10.1212/wnl.27.7.650

9. Omuro AM, Leite CC, Mokhtari K, Delattre JY: Pitfalls in the diagnosis of brain tumours . Lancet Neurol. 2006, 5:937-48. 10.1016/S1474-4422(06)70597-X

10. Grisold W, Oberndorfer S, Struhal W: Stroke and cancer: a review. Acta Neurol Scand. 2009, 119:1-16. 10.1111/j.1600-0404.2008.01059.x

11. Iwama T, Ohkuma A, Miwa Y, et al.: Brain tumors manifesting as intracranial hemorrhage. Neurol Med Chir (Tokyo). 1992, 32:130-5. 10.2176/nmc.32.130

12. Mauriño J, Saposnik G, Lepera S, Rey RC, Sica RE: Multiple simultaneous intracerebral hemorrhages: clinical features and outcome. Arch Neurol. 2001, 58:629-32. 10.1001/archneur.58.4.629

13. Fox BD, Cheung VJ, Patel AJ, Suki D, Rao G: Epidemiology of metastatic brain tumors . Neurosurg Clin N Am. 2011, 22:1-6. 10.1016/j.nec.2010.08.007

14. Suarez-Sarmiento A Jr, Nguyen KA, Syed JS, et al.: Brain metastasis from renal-cell carcinoma: an institutional study. Clin Genitourin Cancer. 2019, 17 :e1163-70. 10.1016/j.clgc.2019.08.006

15. Inohara T, Xian Y, Liang L, et al.: Association of intracerebral hemorrhage among patients taking nonvitamin $\mathrm{k}$ antagonist Vs vitamin $\mathrm{k}$ antagonist oral anticoagulants with in-hospital mortality. JAMA. 2018, 319:463-73. 10.1001/jama.2017.21917 